Check for updates

Cite this: Mater. Chem. Front., 2019, 3, 57

\section{Real time bioimaging for mitochondria by taking the aggregation process of aggregation-induced emission near-infrared dyes with wash-free staining $\dagger$}

\author{
Fei Ren, + Pai Liu $\$$ Yu Gao, Jianbing Shi, (D)* Bin Tong, Zhengxu Cai (D) and \\ Yuping Dong (D) *
}

\begin{abstract}
Fluorescent probing is one of the most powerful methods for bioimaging and/or detecting certain biological species. However, the problem of aggregation-caused quenching in aqueous environments limits the development of high performance fluorophores. In this study, three compounds were designed from triphenylpyrrole (TPP) derivatives with aggregation-induced emission (AIE) characteristics and were applied to real-time mitochondrial imaging. These TPP derivatives emitted near-infrared (NIR) signals by using indolium as an acceptor, both pyrrole and triphenylamine as donors, and ethenylidene as a $\pi$-bridge between the acceptors and donors. The combination of AIE features with NIR emission provides a wash-free procedure for staining the mitochondria in various live cells in less than $10 \mathrm{~s}$. Moreover, the process of signal turn-on could be tracked by taking full advantage of the slow aggregation behavior of the dyes. In addition, the dyes also showed excellent photostabilities under laser irradiation for up to $6 \mathrm{~h}$.
\end{abstract}

Received 24th August 2018 Accepted 19th October 2018

DOI: $10.1039 / c 8 q m 00425 k$

rsc.li/frontiers-materials (4,4-difluoro-4-bora-3a,4a-diaza-s-indacene) fluorophores, diketopyrrolo-pyrrole derivatives, benzothiadiazole and benzobisthiadiazole derivatives. ${ }^{7-9}$ Organic fluorescent molecules that have a large planar $\pi$-conjugated structure may easily form $\pi-\pi$ stacking, which may result in non-radiative decay pathways. This phenomenon occurs in some cases and is called aggregation-caused quenching (ACQ). ${ }^{10}$ ACQ is inevitable in bioimaging and biological detection because biological species normally exist in aqueous environments, and the dyes that are a typical flat molecular structure are insoluble in an aqueous environment, and then aggregate and become non-emissive due to the effective $\pi-\pi$ stacking. Moreover, false signals from ACQ dyes are sometimes detected in real samples due to their complicated biological components. Therefore, the development of anti-ACQ types of fluorescent dyes is necessary. In 2001, Tang's group reported a new compound called 1-methyl-1,2,3,4,5-pentaphenylsilole with aggregation-induced emission (AIE) properties. ${ }^{11}$ The discovery of AIE provides a favorable method for solving the difficult problem of ACQ since a typical AIE molecule, similar to an ACQ one, has a very good $\pi$-conjugation efficiency, but it is not flat, it has a propeller structure. ${ }^{2,12}$ Therefore, its solution is not emissive because of the active rotations or vibration of the molecular units, that non-radiatively deactivate the excited state. Instead in an aggregate or solid state, the molecular movements are impeded, and the only attainable deactivation pathway is the radiative one. In addition, since the molecule does not have a flat shape it will not experience detrimental $\pi-\pi$ stacking in the 
aggregated or solid state. Therefore, the luminescence is turnedon when the molecules are in the aggregated or solid state. If an AIE dye is not well water-soluble, aggregate formation easily occurs in water, and hence AIE dyes are perfect candidates for biological applications where the aqueous environment is the main medium. ${ }^{13-15}$ Excitingly, a series of NIR fluorescent molecules with AIE characteristics have also been designed and effectively used in biological imaging. ${ }^{16}$ These long wavelength emissive materials are designed to lengthen the $\pi$-conjugation of the molecular structure and/or to introduce a strong intramolecular donor-acceptor (D-A) system. ${ }^{17,18}$ For example, Liu's group used 2-(4H-pyran-4-ylidene)malononitrile as an acceptor to prepare a compound with a $\mathrm{D}-\pi-\mathrm{A}$ structure that can be used as a far-red/near-infrared fluorescent probe for in vitro and in vivo imaging; ${ }^{19}$ Liu's group also used benzothiadiazole derivatives to prepare long wavelength excitable NIR fluorescent nanoparticles with aggregation-induced emission characteristics for imageguided tumor resectioning. ${ }^{20}$ Tang's group prepared a kind of AIE nanoparticles with high stimulation emission depletion efficiencies and photobleaching resistances that can be used for long-term super-resolution bio-imaging. ${ }^{21}$ In our recent work about AIE pyrole derivatives with varied D-A structures, we found that different acceptors and different substituted positions on the donors resulted in totally different photophysical properties such as emission wavelength and quantum yield. ${ }^{22}$ Thus, we wanted to know how different donors or donor sizes with different photoluminescent (PL) properties affect their imaging efficiencies. However, a living organism is a very complex environment. Designing a fluorescent dye that not only has long wavelength emissions but also has good imaging effects in living organisms is a significant endeavor. There are no reports regarding the effects of donor size on bio-imaging efficiency.

The cytoplasm of all living cells contains intracellular organelles and bio-macromolecules such as proteins and nucleic acids. ${ }^{23,24}$ These organelles carry out their duties and influence important functions, including metabolism, energy transport and cell proliferation. ${ }^{25-27}$ All these functions require energy, which is produced by mitochondria. As the most important intracellular organelles, mitochondria are associated with many active substances, such as adenosine triphosphate and reactive oxygen species (ROS) during energy metabolism and diseases. ${ }^{28-31}$ Accumulating evidence indicates that ischemiainduced injury is caused by burst production of ROS, and excessive ROS are associated with the possibility of irreversible oxidative damage and carcinogenesis. ${ }^{32,33}$ Any major changes in mitochondrial morphology can be indicative of certain diseases such as cancers or neurodegenerative diseases such as Alzheimer's and Parkinson's diseases. ${ }^{34-36}$ Thus, tracking the changes of mitochondria is significant work that can provide deep insight into energy production, apoptosis and degenerative conditions in clinical research.

Commercial dyes for mitochondrial imaging often suffer certain obvious drawbacks, including small Stokes shift, long incubation periods and numerous washes after cell staining. ${ }^{37-40}$ These long incubation periods (often more than $20 \mathrm{~min}$ ) and numerous washes are long-term unresolved problems. A long incubation often causes nonspecific targeting of cellular components. ${ }^{41}$ Numerous washes are used to improve the signal-to-noise $(\mathrm{S} / \mathrm{N})$ ratio and remove strong residual signals from free dyes. Both processes result in delaying the real-time acquisition of mitochondrial data and decrease the accuracies of cell imaging results. ${ }^{42}$ Though some mitochondrion-targeting AIE probes have been synthesized and used in cell-imaging, most of them are red emitting probes (wavelengths shorter than $700 \mathrm{~nm}$ ). ${ }^{43,44}$ These probes shorten the incubation time from $20 \mathrm{~min}$ to $10 \mathrm{~min}$ and overcome the disadvantages of quenching and multi-washing. However, none of these probes can perform NIR emission (wavelength longer than $700 \mathrm{~nm}$ ) and real-time imaging while also being wash-free and light stable.

With that in mind, we designed a new type of triphenylpyrrole (TPP) derivative with a D-A structure and a mitochondrialtargeting group, as shown in Scheme 1. These derivatives have the following characteristics: (1) the 1-ethyl-3,3-dimethyl-3Hindolium is a strong acceptor and an anchor for the biological target; (2) the triphenylamine (TPA) is a strong donor with or without a spacer linked with the 2,5-position of the pyrrole; (3) the pyrrole group is a weaker donor and a $\pi$-conjugated spacer; (4) the ethyl benzoate group in the 1-position of the pyrrole is a weaker acceptor and a post-functional group. With these configurable variables, three compounds with AIE/aggregation-enhanced emission (AEE) features and deep red/NIR emissions designated TPP-1, TPP-2 and TPP-3 show different PL properties and imaging efficiencies for mitochondria. More importantly, TPP-2 has a very large Stokes shift of up to $219 \mathrm{~nm}$ in solution and $204 \mathrm{~nm}$ in the solid state with a maximum emission wavelength longer than $720 \mathrm{~nm}$. In addition, visual observations for real-time bioimaging of mitochondria were obtained by a wash-free procedure during the incubation period due to the AIE characteristics of these dyes and slow aggregation, the time scale of which is visible to the naked eye in biological matrices. The results of our study indicated that rational D-A structural design and molecular size tuning are effective approaches to obtain high performance NIR AIE dyes that can image real-time processes in biological systems by taking full advantage of their slow aggregation properties.

\section{Results and discussion}

The compounds of interest, TPP-1, TPP-2, and TPP-3, were synthesized by the different routes shown in Scheme S1 (ESI $\dagger$ ).

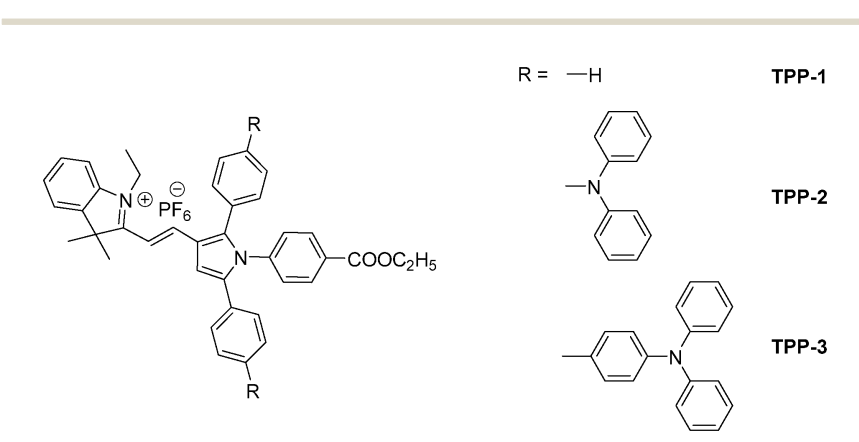

Scheme 1 Chemical structure of target AIE/AEE molecules. 
All the compounds, including intermediates 1-9 and TPP-1-3, were characterized by NMR and MS and the data are in agreement with the intended structure, as shown in Fig. S1-S36 (ESI $\dagger$ ). Moreover, a trans-configuration of TPP-1, TPP-2, and TPP-3 was confirmed by ${ }^{1} \mathrm{H}-{ }^{1} \mathrm{H}$ COSY NMR, as shown in Fig. S37 (ESI $\dagger$ ), due to their coupling constants belonging to the hydrogen of the double bond all being 16.0 Hz. TPP-1, TPP-2, and TPP-3 have good solubilities in polar organic solvents such as dimethyl sulfoxide (DMSO) and $N, N$-dimethyl formamide (DMF), but they have poor solubilities in water due to the tight binding of the positive and negative organic ions and the large hydrophobic aromatic groups. But even with poor partial solubility in water, these TPP derivatives must be in a molecular state when they are at very low concentration due to mutual charge repulsion of their ionic groups.

The photophysical properties of TPP-1, TPP-2 and TPP-3 in solution and in the solid state were investigated after their chemical structures were confirmed, as shown in Fig. 1. As shown in Fig. 1A, the longer absorption bands centered at 467, 514 and $490 \mathrm{~nm}$ for the DMSO solutions of TPP-1, TPP-2 and TPP-3, respectively, are attributed to intramolecular charge transfers from the donors to the acceptors. Notably, the maximum absorption wavelength of TPP-3 was blueshifted compared with that of TPP-2 although its degree of $\pi$-conjugation increased, which might have been caused by the distance between the donor and acceptor and/ or the twisting of the benzene ring that linked with a 1,2,5-position of the pyrrole core. The emission properties of the dyes were different in DMSO solution, as shown in Fig. 1B. TPP-1 showed red emissions with maximum emissive wavelengths of $614 \mathrm{~nm}$, while TPP-2 and TPP-3 showed no emissions except solvent Raman scattering. The time-dependent fluorescence spectra of TPP-1,
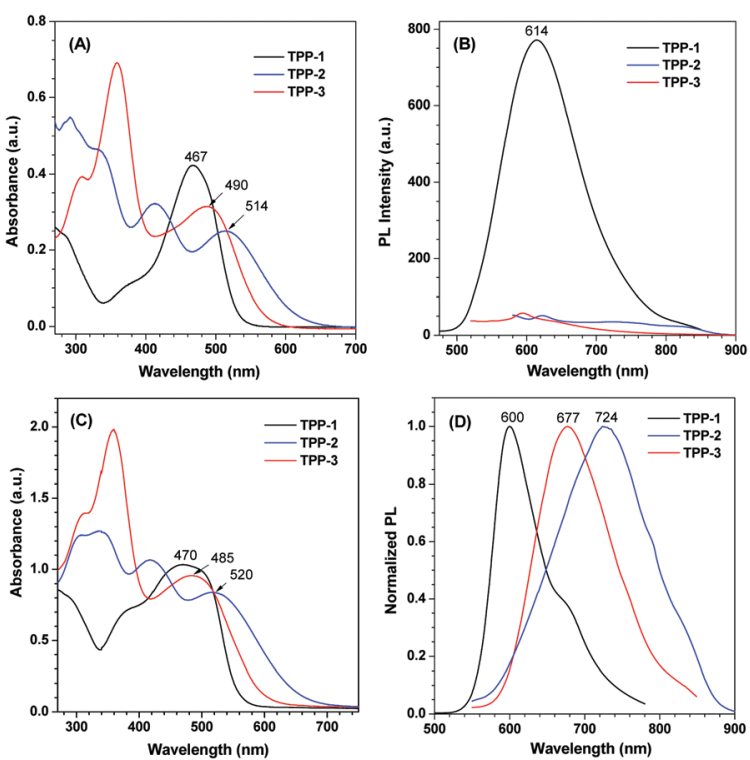

Fig. 1 (A) Absorption spectra of DMSO solutions of TPP-1-3. (B) PL spectra of DMSO solutions of TPP-1-3. (C) Absorption spectra of TPP-1-3 in the solid state. (D) Normalized PL spectra of TPP-1-3 in the solid state. Excitation wavelengths: $\lambda_{\mathrm{ex}}=460 \mathrm{~nm}$ for TPP-1, $520 \mathrm{~nm}$ for TPP-2, and $500 \mathrm{~nm}$ for TPP-3. [TPP-1 $]=[\mathrm{TPP}-2]=[\mathrm{TPP}-3]=1.0 \times 10^{-5} \mathrm{~mol} \mathrm{~L}^{-1}$.
TPP-2 and TPP-3 showed that these DMSO solutions are very stable (Fig. S38, ESI $\dagger$ ). Such a different emissive behavior in solution made us eager to know whether there was a difference in the solid state. Thus, we measured the photophysical properties of the dyes in the solid state, as shown in Fig. 1C and D. Their UV-Vis spectra in the solid state are similar to those in solution (Fig. 1C), but the maximum emission wavelength of TPP-2 in the solid state is much longer than those of TPP-1 and TPP-3 (Fig. 1D). Similar results were obtained in DMSO solution or the solid state even at the same excitation wavelength of $500 \mathrm{~nm}$ (Fig. S39, ESI $\dagger$ ). In general, the higher the degree of conjugation, the longer the emission wavelength under the same donors and acceptors. ${ }^{45}$ However, our results indicated that the charge transfer distances between donors and acceptors were very important in the design of NIR emissive dyes.

Next, we studied the aggregation behaviors of TPP-1, TPP-2 and TPP-3 in mixtures of a good solvent (DMSO) and a poor solvent (water) with different water fractions $\left(f_{\mathrm{w}}\right)$, as shown in Fig. S40 (ESI $\dagger$ ). The net changes of the maximum PL intensities with different $f_{\mathrm{w}}$ are summarized in Fig. 2. Although there is a cationic group in each compound, their water solubilities are poor because of both the large aromatic structures of and tight ionic pairing between organic indolium and hexafluorophosphate. When $f_{\mathrm{w}}$ increased from 0 to $90 \mathrm{vol} \%$, the PL intensity of TPP-1 decreased slightly due to the solvent polarity change. However, the PL intensity of TPP-1 significantly increased when $f_{\mathrm{w}}$ reached 99 vol\%, which was caused by TPP-1 aggregation due to the high fraction of poor solvent. Dynamic light scattering (DLS) confirmed aggregate formation with TPP-1 particle diameters of 1.2, 23.5 and $168.1 \mathrm{~nm}$ at $f_{\mathrm{w}}=0,90$ and $99 \mathrm{vol} \%$, respectively, as shown in Fig. S41A-C (ESI $\dagger$ ). For TPP-2, the maximum PL intensity increased greater than 14 -fold when $f_{\mathrm{w}}$ reached 70 vol\%, which was the highest enhanced efficiency for the three compounds. The enhancement of TPP-3 was greater than 12-fold when $f_{\mathrm{w}}$ reached 60 vol\%. Due to an increasing number of hydrophobic parts in these molecules, smaller fractions of water can force them to aggregate. The DLS results for TPP-2 and TPP-3 also confirmed the formation of aggregates, as shown in Fig. S41D-G (ESI $\dagger$ ). Moreover, tracking the UV-visible spectra showed little intensity changes in the visible region when adding water into the solution, as shown in Fig. S42 (ESI $\dagger$ ),

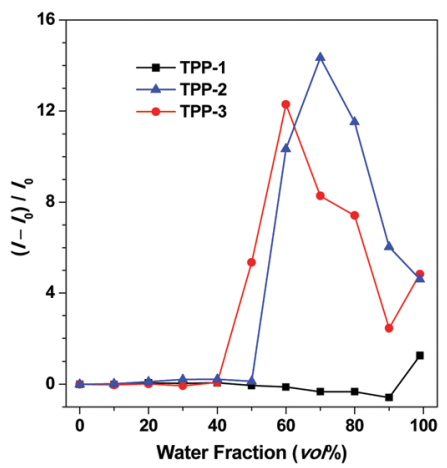

Fig. 2 Net changes in the maximum PL intensities of TPP-1-3 in different $f_{\mathrm{w}} \cdot I_{0}$ is the PL intensity at $f_{\mathrm{w}}=0$. 
which is an evidence of aggregate formation. In addition, SEM images also identified these aggregates in the mixture of $\mathrm{DMSO} / \mathrm{H}_{2} \mathrm{O}$, as shown in Fig. S43 (ESI $\dagger$ ). These results indicate that TPP-1 is an AEE molecule but both TPP-2 and TPP-3 are AIE molecules. The color change was obviously observed in the photographs of their corresponding solution except TPP-2 that is NIR emission, as shown in Fig. S44 (ESI $\dagger$ ). Among them, TPP-2 shows the longest emission wavelength, up to the NIR region, and the most enhanced PL maximum intensity, up to 14-fold, that is, TPP-2 shows good NIR PL behaviors and AIE features.

For deep insight into their unique PL and AIE properties, we tried to obtain single crystals of TPP-1, TPP-2 and TPP-3 to clarify the compact arrangements of their aggregates. However, only one single crystal, TPP-1, was grown by slowly evaporating a solvent mixture of dichloromethane/ethyl acetate (1:1). As shown in Fig. 3, the triphenylpyrrole derivative exhibited a very twisted conformation with dihedral angles of $65.0^{\circ}, 56.7^{\circ}$ and $37.9^{\circ}$ between the pyrrole ring and the benzene rings on its 1,2,5-position (Fig. 3A), which prevented intermolecular $\pi-\pi$ interactions that reduced fluorescence emissions. Moreover, intermolecular $\mathrm{C}-\mathrm{H} \cdots \mathrm{O}$ interactions were observed in the crystal packing structure (Fig. 3B), which can also improve the degree of the restriction of intramolecular rotation (RIR) and further block the non-radiative release of energy and thus advantageously enhanced the PL efficiency in the aggregated state. ${ }^{46}$ The packing of the molecule in the crystal structure has been checked and it is shown in Fig. 3C and D. It was found that molecular units were continually expanded to form tight molecular layers of the crystal. All these factors are beneficial for fluorescence emission in the aggregated state. ${ }^{47}$ Thus, the particular molecular conformation and intermolecular packing of TPP-1 ensured its AEE features in the aggregation state. On the other hand, the torsion angle between the indolium ring and the pyrrole ring through the $\pi$-bridge of ethenylidene is only $13.9^{\circ}$, which indicates good coplanar conformation in this conjugated structure. This coplanarity is beneficial for the charge transfer in the $\mathrm{D}-\mathrm{A}$ structure and results in longer wavelength emissions. Moreover, the optimized structure of TPP-1 calculated by using the B3LYP/6-31G basis set with the

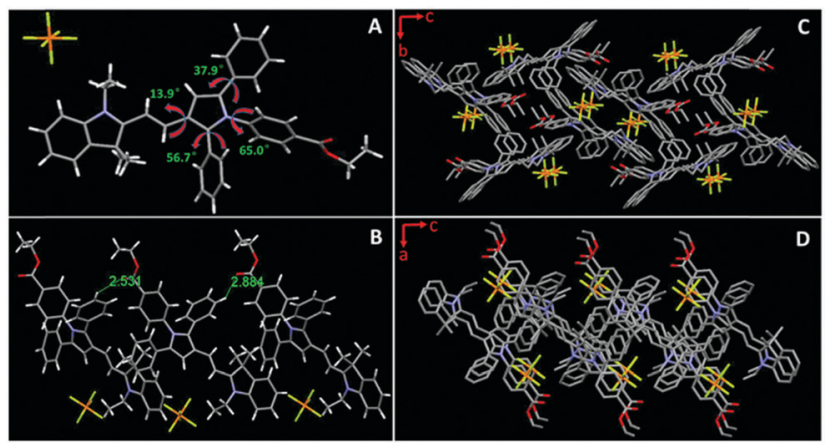

Fig. 3 Single crystal structure of TPP-1 (CCDC 1853627†): (A) molecular structure, (B) intermolecular interactions, and (C and D) molecular packing mode (hydrogen omitted forclarity).
G09 program was similar to the crystal structure, with angles of $62.6^{\circ}, 68.0^{\circ}$ and $43.2^{\circ}$ between the pyrrole ring and the benzene rings on its $1,2,5$-position and $10.5^{\circ}$ between the indolium ring and the pyrrole ring, as shown in Fig. S45A (ESI $\dagger$ ). Although we cannot obtain single crystals of the other compounds TPP-2 and TPP-3, the optimized geometries of TPP-2 and TPP-3 were similar to each other and similar to TPP-1 as the following dihedral angles between the pyrrole/phenyl (1,2,5-position of pyrrole) ring and the pyrrole/indolium ring were found: $65.2^{\circ}$, $55.4^{\circ}, 40.3^{\circ}$ and $13.6^{\circ}$ for $\mathbf{T P P}-2$, and $62.8^{\circ}, 64.0^{\circ}, 41.9^{\circ}$ and $12.8^{\circ}$ for TPP-3, respectively, as shown in Fig. S45B and C (ESI $\dagger$ ). So we can infer that both TPP-2 and TPP-3 might adopt similar conformations to TPP-1 in the aggregated state because they have similar chemical structures and optimized molecular dihedral angles of these rings. It is noteworthy that there is a little difference of the twisted angles between the pyrrole ring and the phenyl ring that linked the 2-position of the pyrrole, which are $68.0^{\circ}$ for TPP-1, 55.4 ${ }^{\circ}$ for TPP-2 and $64.0^{\circ}$ for TPP-3, probably due to the crowed chemical structure environment. The smaller twisting angle means better ability of charge transfer, which is advantageous for longer emission. The conjugation degree is more enhanced in TPP-3 because of an extra phenyl ring; however, this also confers a higher twisted angle and then cancels the benefit of the increased $\pi$-conjugation. This is in agreement with the abovementioned photophysical properties explaining why TPP-3 compared to TPP-2 has bluer absorption and emission spectra. All abovementioned results demonstrated that rational design of NIR dyes could be realized by balancing the donation ability with the real transfer distance in D-A structural AIE/AEE dyes and adjusting the twisted angles of the D/A moieties.

The fluorescence lifetime $(\tau)$ and quantum yield $(\Phi)$ are the main parameters for emissive dyes. To quantitatively evaluate the obtained TPP emissions, we first measured the lifetime of TPP-1, TPP-2 and TPP-3 in DMSO but only got the value of TPP-1, which was 1.05 ns, since TPP-2 and TPP-3 are nonemissive in solution (Table S1, ESI $\dagger$ ). Then, we investigated the fluorescence lifetimes of TPP-1, TPP-2 and TPP-3 in the solid state and in a mixture of DMSO/water in an aggregated state (Table S2, ESI $\dagger$ ). Moreover, their corresponding quantum yields were obtained by using an integrating sphere. All data are summarized in Table 1. According to the equations $\tau=1 /\left(k_{\mathrm{r}}+k_{\mathrm{nr}}\right)$ and $\Phi=k_{\mathrm{r}} /\left(k_{\mathrm{r}}+k_{\mathrm{nr}}\right)$, where $k_{\mathrm{r}}$ is the radiative rate of the dye, and $k_{\mathrm{nr}}$ is its rate of nonradiative decay, their $k_{\mathrm{r}}$ and $k_{\mathrm{nr}}$ were calculated and are also presented in Table 1 . The fluorescence decay curves revealed that TPP-2 has a shorter lifetime than TPP-3, whether they are in the aggregated or solid state, as shown in Fig. S46 (ESI $\dagger$ ). The quantum yields of TPP-1, TPP-2 and TPP-3 in their aggregate forms are $1.33 \%, 0.19 \%$, and $0.05 \%$, respectively, and those in their solid states are $8.61 \%, 1.13 \%$ and $1.86 \%$, respectively. The quantum yields in the solid states are slightly higher than those in the aggregate states because there is no water solvent, which greatly reduces the emission efficiency, and they have a more compact arrangement that is beneficial for RIR. The data for $k_{\mathrm{nr}}$ confirmed this trend because all the values for $k_{\mathrm{nr}}$ in the aggregates were higher than those in the respective solids. 
Table 1 The lifetime and quantum yield of TPP-1-3 in aggregates and in the solid state

\begin{tabular}{|c|c|c|c|c|c|c|c|c|}
\hline \multirow[b]{2}{*}{ Compound } & \multicolumn{2}{|c|}{$\begin{array}{l}\text { Lifetime } \\
\tau \text { (ns) }\end{array}$} & \multicolumn{2}{|c|}{$\begin{array}{l}\text { Quantum } \\
\text { yield } \Phi(\%)\end{array}$} & \multicolumn{2}{|c|}{$\begin{array}{l}\text { Radiative rate } \\
k_{\mathrm{r}}\left(\times 10^{7} \mathrm{~s}^{-1}\right) \\
\end{array}$} & \multicolumn{2}{|c|}{$\begin{array}{l}\text { Nonradiative rate } \\
k_{\mathrm{nr}}\left(\times 10^{8} \mathrm{~s}^{-1}\right)\end{array}$} \\
\hline & Aggr. & Solid & Aggr. & Solid & Aggr. & Solid & Aggr. & Solid \\
\hline TPP-1 & 0.75 & 1.30 & 1.33 & 8.61 & 1.773 & 6.623 & 13.156 & 7.030 \\
\hline TPP-2 & 0.65 & 0.89 & 0.19 & 1.13 & 0.2923 & 1.270 & 15.355 & 11.109 \\
\hline TPP-3 & 1.25 & 1.49 & 0.05 & 1.86 & 0.0400 & 1.248 & 7.996 & 6.587 \\
\hline
\end{tabular}

Note: Aggr. is the abbreviation for aggregates made from mixtures of DMSO and water at $f_{\mathrm{w}}=99 \mathrm{vol} \%$ for TPP-1, $f_{\mathrm{w}}=70 \mathrm{vol} \%$ for TPP-2, and $f_{\mathrm{w}}=60 \mathrm{vol} \%$ for TPP-3. $[\mathbf{T P P}-1]=[\mathbf{T P P}-2]=[\mathbf{T P P}-3]=1.0 \times 10^{-5} \mathrm{~mol} \mathrm{~L}^{-1}$.

For bioimaging or detection in an aqueous environment, a turn-on signal is desirable to avoid false signals. Therefore, NIR emissive dyes of TPP derivatives whose emission is turned on in an aqueous environment, are ideal materials for bioprobes due to their AIE characteristics and large Stokes shifts.

Cytotoxicity is an important assay for applications in living cell imaging and in vivo imaging. Thus, to determine the cytotoxic effects of probes TPP-1, TPP-2 and TPP-3, we carried out an apoptosis assay experiment on human cervical cancer cells (HeLa). As shown in Fig. S47 (ESI $\dagger$ ), almost 95\% of the HeLa cells survived after $48 \mathrm{~h}$ of co-staining. No obvious early apoptosis or death of the HeLa cells was observed after 12, 24, and $48 \mathrm{~h}$ incubation periods with $1.0 \times 10^{-7} \mathrm{~mol} \mathrm{~L}^{-1}$ of TPP-1, TPP-2 or TPP-3, which indicated that these probes are suitable for bio-imaging.

To evaluate the application and tracking in live cells, HeLa cells were used as test candidates. We found that TPP-1, TPP-2 and TPP-3 were all cell permeable and specifically stained mitochondria but showed different imaging effects. As shown in Fig. S48 (ESI $\dagger$ ), TPP-1 and TPP-2 were present in similar amounts in HeLa cells after the same incubation times, but a lower amount of TPP-3 was present, indicating that molecular size is a key factor for in vivo bioimaging. In order to further confirm the size of TPP-1, TPP-2 and TPP-3 in DMEM, DLS was used and the results of 5.3, 6.9 and $11.1 \mathrm{~nm}$ were obtained, as shown in Fig. S49 (ESI $\dagger$ ), which had no aggregates in DMEM. Moreover, there is no interference of ionic species on their emission in the cell staining matrix, with $\mathrm{NaCl}$ as an example shown in Fig. S50 (ESI $\dagger$ ). As shown in Fig. S51 (ESI $\dagger$ ), TPP-1 and TPP-2 showed brighter imaging effects than TPP-3 because they are smaller compared to TPP-3 and easily accumulated in larger amounts in HeLa cells, and TPP-3 was the last choice for bioimaging due to having the largest rigid molecular structure, which is detrimental for cytophagy. Thus, TPP-2 was the best choice for mitochondrial imaging due to its longer emission wavelength from the viewpoint that a redder emission wavelength can increase the signal-to-noise ratio and decrease the background, which further could be more suitable for imaging in vivo. ${ }^{34,43,44}$ The specific targeting was mainly attributed to both its positively charged characteristic indolium group and the amphiphilic property of the whole molecule. ${ }^{43}$ To determine the specific targeting component, a co-localization experiment was performed with commercial Mitotracker Green, a green
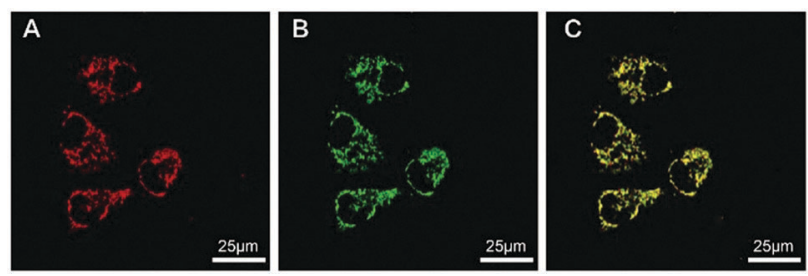

Fig. 4 Confocal images of HeLa cells stained with (A) TPP-2 $(1.0 \times$ $\left.10^{-7} \mathrm{~mol} \mathrm{~L}^{-1}\right)$, (B) MitoTracker Green $\left(1.0 \times 10^{-7} \mathrm{~mol} \mathrm{~L}^{-1}\right)$, and (C) merged.

fluorescent dye that targets mitochondria. The HeLa cells display bright red fluorescence when co-stained by TPP-2 and green fluorescence from Mitotracker Green, as shown in Fig. 4. The merged images indicated that the distribution of TPP-2 in the HeLa cells was almost consistent with that of Mitotracker Green, which proved the specific selectivity of TPP-2 to mitochondria.

To evaluate the wider applicability for targeting mitochondria, we further assessed the abilities of TPP-1, TPP-2, and TPP-3 in different cell lines, such as a human liver cancer cell line (HepG2), a mouse embryo fibroblast cell line (NIH 3T3), and a human breast carcinoma cell line (MCF-7). The tested cell lines were treated with TPP-1, TPP-2, and TPP-3 at concentrations of $1.0 \times 10^{-7} \mathrm{~mol} \mathrm{~L}^{-1}$ for $5 \mathrm{~min}$. As shown in Fig. S52-S54 $(\mathrm{ESI} \dagger)$, different fluorescence intensities were observed in the tested cells, and all the results demonstrated that TPP-1 and TPP-2 had similar fluorescence intensities. These results demonstrated that TPP-2 could be used for the bio-imaging of mitochondria in different cell lines. Furthermore, the photostability of TPP-2 was excellent even after continuous irradiation for over $6 \mathrm{~h}$, as shown in Fig. S55 (ESI $\dagger$ ), while that of Mitotracker Red decreased greatly after $1 \mathrm{~h}$ at the same concentration and laser intensity. These results indicated that our NIR AIE probe had a higher photostability than those of commercial dyes, which is advantageous for tracking long term biological processes.

For live cell imaging, real-time tracking of a special organelle and its relevant processes is still challenging because of complex biological matrices. ${ }^{48,49}$ Most dyes aggregate in biological environments and result in fluorescence changes themselves. However, for AIE dyes, aggregation is a natural advantage because the more aggregated the molecules, the more intense the dye. According to the aforementioned results, the final dyeing effect differs with structural size when imaging HeLa cells by co-staining with TPP-1, TPP-2 or TPP-3 for 5 min. As shown in Fig. 5 and the corresponding videos (ESI $\dagger$ ), we recorded the incubation processes and obtained real-time accumulating moments. To our surprise, the different dyes showed very different staining times even without a washing procedure. TPP-1 quickly targets mitochondria in less than $5 \mathrm{~s}$, TPP-2 has a similar effect at approximately $10 \mathrm{~s}$, and TPP-3 shows no obvious fluorescence even after $30 \mathrm{~s}$ because of its large structural size. The mitochondria can be clearly imaged with the help of TPP-2 with its redder wavelength and excellent image contrast against the cell background after $10 \mathrm{~s}$. More importantly, the aggregation process also perfectly presents in a distinguished time interval under the simplest staining steps, which would be very useful for tracking certain physiological processes of specific 


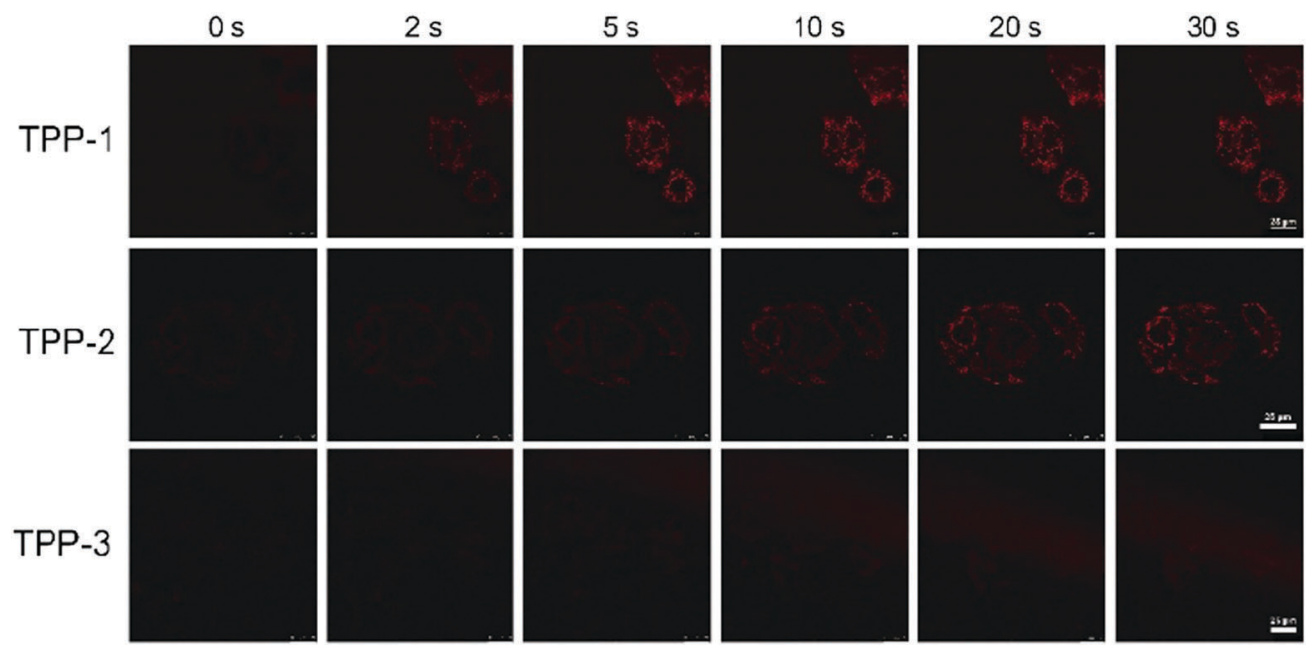

Fig. 5 Screen shot from the real-time video after addition of TPP-1, TPP-2 and TPP-3 into HeLa cells with the same conditions of [TPP-1] $=[$ TPP-2] = $[\mathrm{TPP}-3]=1.0 \times 10^{-7} \mathrm{~mol} \mathrm{~L}^{-1}$.

cells and getting the most accurate cell information. Due to its NIR emission with AIE characteristics and photostable performance, TPP-2 has the potential to realize real-time imaging of mitochondria on site, which will probably reveal some interesting truths regarding mitochondria-related behaviors.

\section{Conclusions}

In summary, three pyrrole derivatives with differently structured donors showed different photophysical properties. The AIE and NIR characteristics of TPP-2 show excellent performance in the bioimaging of special components in complex biological systems. TPP-2 can be used for real-time imaging of mitochondria by taking full advantage of its special AIE properties; the more aggregated it is, the brighter it is. The staining process can be performed by a washing-free procedure at the low concentration of $10^{-7} \mathrm{~mol} \mathrm{~L}^{-1}$ in less than $10 \mathrm{~s}$. Moreover, TPP-2 has very good photostability during long, continuous irradiations of up to $6 \mathrm{~h}$. In addition, real-time imaging of mitochondria in most common cells can also be realized by using the aggregation process of TPP-2 and turn-on signals in biological matrices. The results indicate that rationally designing pyrrole derivatives can provide promising bioprobes with AIE features in the NIR region for realtime bioimaging, and the post-functionality of ethyl benzoate on the 1-position of TPP derivatives also offers more options for biological applications. More potential applications are under investigation in our lab and our collaborators' lab.

\section{Conflicts of interest}

There are no conflicts to declare.

\section{Acknowledgements}

This work was financially supported by the National Natural Science Foundation of China (Grants 21474009, 51673024 and
21490574) and Graduate Technological Innovation Project of Beijing Institute of Technology (2018CX10005).

\section{Notes and references}

1 H. J. Chen, C. Y. Chew, E. H. Chang, Y. W. Tu, L. Y. Wei, B. H. Wu, C. H. Chen, Y. T. Yang, S. C. Huang, J. K. Chen, I. C. Chen and K. T. Tan, J. Am. Chem. Soc., 2018, 140, 5224.

2 J. Mei, Y. Hong, J. W. Y. Lam, A. Qin, Y. Tang and B. Z. Tang, Adv. Mater., 2014, 26, 5429.

3 J. J. Liu, J. Yang, J. L. Wang, Z. F. Chang, B. Li, W. T. Song, Z. Zhao, X. Lou, J. Dai and F. Xia, Mater. Chem. Front., 2018, 2, 1126.

4 M. Gao, F. Yu, C. Lv, J. Choo and L. Chen, Chem. Soc. Rev., 2017, 46, 2237.

5 Q. Yang, Z. Hu, S. Zhu, R. Ma, H. Ma, Z. Ma, H. Wan, T. Zhu, Z. Jiang, W. Liu, L. Jiao, H. Sun, Y. Liang and H. Dai, J. Am. Chem. Soc., 2018, 140, 1715.

6 X. Cai, J. Liu, W. H. Liew, Y. Duan, J. Geng, N. Thakor, K. Yao, L. D. Liao and B. Liu, Mater. Chem. Front., 2017, 1, 1556.

7 B. Li, L. Lu, M. Zhao, Z. Lei and F. Zhang, Angew. Chem., Int. Ed., 2018, 57, 7483.

8 Q. Yang, Z. Ma, H. Wang, B. Zhou, S. Zhu, Y. Zhong, J. Wang, H. Wan, A. Antaris, R. Ma, X. Zhang, J. Yang, X. Zhang, H. Sun, W. Liu, Y. Liang and H. Dai, Adv. Mater., 2017, 29, 1605497.

9 J. Miao, Y. Huo, H. Shi, J. Fang, J. Wang and W. Guo, J. Mater. Chem. B, 2018, 6, 4466.

10 R. T. Kwok, C. W. Leung, J. W. Y. Lam and B. Z. Tang, Chem. Soc. Rev., 2015, 44, 4228.

11 J. Luo, Z. Xie, J. W. Y. Lam, L. Cheng, B. Z. Tang, H. Chen, C. Qiu, H. S. Kwok, X. Zhan, Y. Liu and D. Zhu, Chem. Commun., 2001, 1740.

12 Z. Peng, Y. Ji, Z. Huang, B. Tong, J. Shi and Y. Dong, Mater. Chem. Front., 2018, 2, 1175. 
13 G. Liu, D. Chen, L. Kong, J. Shi, B. Tong, J. Zhi, X. Feng and Y. Dong, Chem. Commun., 2015, 51, 8555.

14 P. Liu, D. Chen, Y. Wang, X. Tang, H. Li, J. Shi, B. Tong and Y. Dong, Biosens. Bioelectron., 2017, 92, 536.

15 D. Chen, H. Wang, P. Liu, L. Song, J. Shi, B. Tong and Y. Dong, Anal. Chim. Acta, 2018, 1001, 151.

16 S. He, J. Song, J. Qu and Z. Cheng, Chem. Soc. Rev., 2018, 47, 4258.

17 W. Qin, P. Zhang, H. Li, J. W. Y. Lam, Y. Cai, R. T. K. Kwok, J. Qian, W. Zheng and B. Z. Tang, Chem. Sci., 2018, 9, 2705.

18 Y. Zhang, W. Xu, L. Kong, B. Han, Z. Cai, J. Shi, B. Tong, Y. Dong and B. Z. Tang, Mater. Chem. Front., 2018, 2, 1779.

19 W. Qin, D. Ding, J. Liu, W. Z. Yuan, Y. Hu, B. Liu and B. Z. Tang, Adv. Funct. Mater., 2012, 22, 771.

20 J. Liu, C. Chen, S. Ji, Q. Liu, D. Ding, D. Zhao and B. Liu, Chem. Sci., 2017, 8, 2782.

21 D. Li, W. Qin, B. Xu, J. Qian and B. Z. Tang, Adv. Mater., 2017, 29, 1703643.

22 Y. Lei, Y. Lai, L. Dong, G. Shang, Z. Cai, J. Shi, J. Zhi, P. Li, X. Huang, B. Tong and Y. Dong, Chem. - Eur. J., 2018, 24, 434.

23 R. Guo, J. Yin, Y. Ma, Q. Wang and W. Lin, J. Mater. Chem. B, 2018, 6, 2894.

24 G. Guigas, C. Kalla and M. Weiss, Biophys. J., 2007, 93, 316.

25 X. Li, J. Kim, J. Yoon and X. Chen, Adv. Mater., 2017, 29, 1606857.

26 Y. P. Ow, D. R. Green, Z. Hao and T. W. Mak, Nat. Rev. Mol. Cell Biol., 2008, 9, 532.

27 F. M. Yakes and B. V. Houten, Proc. Natl. Acad. Sci., 1997, 94, 514.

28 P. D. Ray, B. W. Huang and Y. Tsuji, Cell. Signalling, 2012, 24, 981.

29 M. Valko, D. Leibfritz, J. Moncol, M. T. Cronin, M. Mazur and J. Telser, Int. J. Biochem. Cell Biol., 2007, 39, 44.

30 I. Ohsawa, M. Ishikawa, K. Takahashi, M. Watanabe, K. Nishimaki, K. Yamagata, K. Katsura, Y. Katayama, S. Asoh and S. Ohta, Nat. Med., 2007, 13, 688.

31 N. Alifu, X. Dong, D. Li, X. Sun, A. Zebibula, D. Zhang, G. Zhang and J. Qian, Mater. Chem. Front., 2017, 1, 1746.
32 M. Gao, R. Wang, F. Yu, J. You and L. Chen, J. Mater. Chem. $B, 2018,6,2608$.

33 R. D. Guzy, B. Hoyos, E. Robin, H. Chen, L. Liu, K. D. Mansfield, M. C. Simon, U. Hammerling and P. T. Schumacker, Cell Metab., 2005, 1, 401.

34 J. Zou, H. Lu, X. Zhao, W. Li, Y. Guan, Y. Zheng, L. Zhang and H. Gao, Dyes Pigm., 2018, 151, 45.

35 D. M. Hockenbery, Environ. Mol. Mutagen., 2010, 51, 476.

36 S. Gandre-Babbe and A. M. van der Bliek, Mol. Biol. Cell, 2008, 19, 2402.

37 J. Zhao, W. Wu, J. Sun and S. Guo, Chem. Soc. Rev., 2013, 42, 5323.

38 C. Zhang, S. Jin, K. Yang, X. Xue, Z. Li, Y. Jiang, W. Q. Chen, L. Dai, G. Zou and X. J. Liang, ACS Appl. Mater. Interfaces, 2014, 6, 8971.

39 H. Shi, J. Liu, J. Geng, B. Z. Tang and B. Liu, J. Am. Chem. Soc., 2012, 134, 9569.

40 Q. Y. Jiang, L. H. Lai, J. Shen, Q. Q. Wang, F. J. Xu and G. P. Tang, Biomaterials, 2011, 32, 7253.

41 M. D. Shi, J. K. Chao, M. C. Ma, S. K. Chiang and I. C. Chao, Int. J. Biochem. Cell Biol., 2014, 26, 235.

42 M. E. Lidell, M. J. Betz, O. Dahlqvist Leinhard, M. Heglind, L. Elander, M. Slawik, T. Mussack, D. Nilsson, T. Romu, P. Nuutila, K. A. Virtanen, F. Beuschlein, A. Persson, M. Borga and S. Enerback, Nat. Med., 2013, 19, 631.

43 J. Li, N. Kwon, Y. Jeong, S. Lee, G. Kim and J. Yoon, ACS Appl. Mater. Interfaces, 2018, 10, 12150.

44 N. Zhao, S. Chen, Y. Hong and B. Z. Tang, Chem. Commun., 2015, 51, 13599.

45 S. Xu, W. Wu, X. Cai, C. J. Zhang, Y. Yuan, J. Liang, G. Feng, P. Manghnani and B. Liu, Chem. Commun., 2017, 53, 8727.

46 J. Mei, N. L. Leung, R. T. Kwok, J. W. Y. Lam and B. Z. Tang, Chem. Rev., 2015, 115, 11718.

47 C. Li, R. Duan, B. Liang, G. Han, S. Wang, K. Ye, Y. Liu, Y. Yi and Y. Wang, Angew. Chem., Int. Ed., 2017, 56, 11525.

48 B. Situ, S. Chen, E. Zhao, C. W. T. Leung, Y. Chen, Y. Hong, J. W. Y. Lam, Z. Wen, W. Liu, W. Zhang, L. Zheng and B. Z. Tang, Adv. Funct. Mater., 2016, 26, 7132.

49 Y. Guan, H. Lu, W. Li, Y. Zheng, Z. Jiang, J. Zou and H. Gao, ACS Appl. Mater. Interfaces, 2017, 9, 26731. 